\title{
Reliability-based Optimum Design of Glulam Cable-Stayed Footbridges $1^{\mathrm{s} i t}$ TDregf
}

\author{
João.H.O. Negrão and Luis M.C. Simões
}

Dept. Civil Engineering, University of Coimbra, Portugal, jhnegrao@dec.uc.pt, lcsimoes@ dec.uc.pt

\begin{abstract}
This work presents a procedure for finding the reliability-based optimum design of cable stayed bridges. The minimization problem is stated as the minimization of stresses, displacements, reliability and bridge cost. A finiteelement approach is used for structural analysis. It includes a direct analytic sensitivity analysis module, which provides the structural behaviour responses to changes in the design variables. An equivalent multicriteria approach is used to solve the non-differentiable, non-linear optimization problem, turning the original problem into the sequential minimization of unconstrained convex scalar functions, from which a Pareto optimum is obtained. Examples are given illustrating the procedure.
\end{abstract}

Keywords: Structural optimization, Cable-stayed bridge, Reliability-based design, Multiple-objectives

\section{Introduction}

The optimization of cable-stayed bridges can be stated as that of the minimization of structural cost or volume, and the maximum stresses throughout the structure. Additional objectives are aimed at the deflections or displacements and to guarantee that the design variables are at least specified minimum values. The work started with the shape and sizing optimization by using a 2D finite-element model for the analysis. The problem was extended to three-dimensional analysis and the consideration of erection stages under static loading (Negrão and Simões, 1997). Seismic effects were considered in the optimization both by a modal-spectral approach and a time-history based procedure (Simões and Negrão, 1999). In most of the previous studies, a grid solution was adopted for modelling the deck, with stiffening girders supporting transverse beams, although box-girder sections were employed (Negrão and Simões, 1999). Prestressing design variables were also considered for the problems of optimal correction of cable forces during erection. Deterministic Optimization enhanced by reliability performance and formulated within the probabilistic framework is called reliability-based optimum design. These are considered important ingredients in the design of advanced structural systems. Wider applications still exhibit limitations mainly attributed to the deeply nested architecture of this procedures involving analysis with finite elements, reliability analysis, sensitivity analysis and optimization.

The behaviour of complex structures is often analysed by means of Finite Element Analysis (FEA). Stresses and deformations of the structure can be computed given the (deterministic) parameters of loads, geometry and material behaviour. Some structural codes specify a maximum probability of failure within a given reference period (lifetime of the structure). This probability of failure is ideally translated into partial safety factors and combination factors by which variables like strength and load have to be divided or multiplied to find the so-called design values. These design values are to be used as input for a Finite Element Analysis. The outcome of the calculations is compared with the limit states (for example collapse or maximum deformation). The structure is supposed to have met the reliability requirements when the limit states are not exceeded. Reality is different. First of all the (code type) level I method using partial safety factors makes it only plausible that the reliability requirements are met for average structures. Second aspect is that safety factors are often based on experience only. A link with the required reliability on a theoretical basis often does not exist. Third aspect is the system behaviour of structures. The safety factors are often derived for components of the structure like girders and columns. A structure as a whole behaves like a system of these components. As a result, depending on the kind of system, the structure can be more or less reliable than its components. The advantage of the code type level I method (using partial safety factors out of codes) is that, the limit states are to be checked (by means of an FEA) for only a relatively small number of combinations of variables. A disadvantage is the lack of accuracy. A code type level I method uses partial safety factors which lead to sufficient reliability for average components of structures. These problems can be overcome by using more sophisticated reliability methods such as level II (FORM) and level III (Monte Carlo) reliability methods. The problem with these methods is the considerable computational effort when used in combination with FEA. The combination of Directional Sampling and a Response Surface method is a kind of importance directional sampling using a smaller number of limit state evaluations. The improvement to the standard directional sampling procedure lies in the use of FEA for the important directions and a response surface for less important directions. In practise this means that, after the response surface is constructed, only few FEA computations have to be performed.

In this work first order reliability methods were used and the sensitivity information was obtained analytically. In a forthcoming paper the advanced simulation method combined with the response surface method will be proposed. 


\section{Structural Analysis}

The finite element based open code MODULEF was used as the basic tool for structural analysis, because code availability was a fundamental requirement for further developments. Out of the several element types included in the element library of the programme, only the FE required for two- and three-dimensional models of cable-stayed bridges were retained and adapted to specific needs. These were 2D and 3D bar and beam (Euler-Bernoulli formulation) elements and 4- and 8-noded serendipity plate-membrane (Reissner-Mindlin formulation) elements.

\section{Design Variables}

The structural response of a cable-stayed bridge is conditioned by a large number of parameters, concerning crosssectional shapes and dimensions, overall bridge geometry, applied prestressing forces, deck-to-pylon connections, etc. Some of them play only a limited role on the bridge behaviour while others, such as the cable pattern and prestressing forces, are of major importance for both the safety and serviceability purposes. Three types of design variables were considered: sizing, shape and mechanical. Sizing design variables are cross-sectional characteristics of bar, beam and plate elements, such as web height, flange width, plate thickness, etc. Changes of such variables do not imply the need for remeshing. Shape design variables produce geometry changes that require nodal co-ordinates update or even complete remeshing. Other design variables can be characterized as hybrid, because they define both the box-girder cross-section shape and the deck geometry, requiring co-ordinates updating only. Finally, the fixed-end prestressing force is a mechanical design variable not related to any geometric quantity. The currently available types are shown in Figure 1.

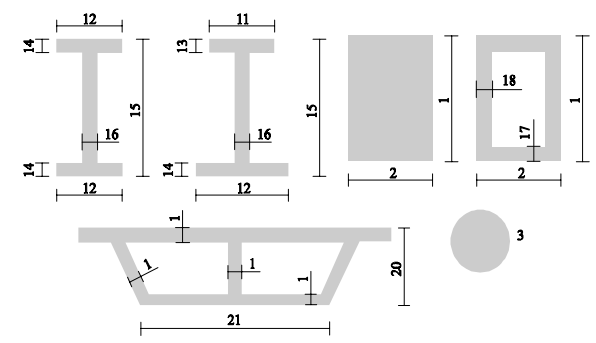

Fig. 1 - Sizing and hybrid design variables

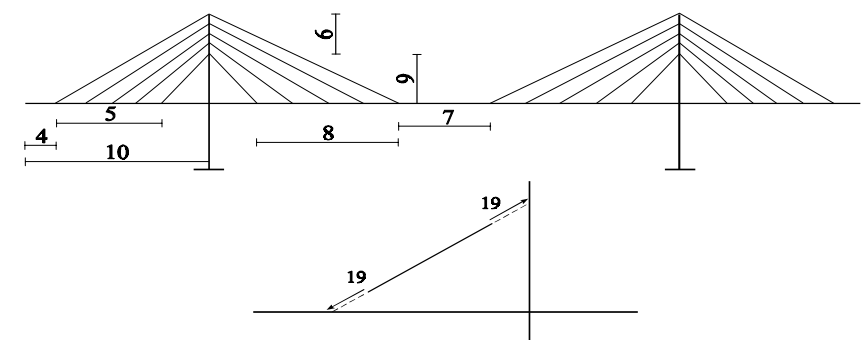

Fig. 2 - Shape and mechanical design variables

All these types play complementary roles in the process of design optimization. Sizing design variables directly provide for cost/volume decrease. Shape and mechanical design variables have a neglectable direct relation to structural cost but allow for better stress distributions, which in turn lead to further decreases in sizing variables. Prestressing force design variables are essential for achieving acceptable solutions when deflections are considered in the dead load condition.

The final behaviour of cable-stayed bridges is deeply related to the erection. Among the various methods used for bridge erection, cantilevering method has become the most popular, due to its suitability for building large spans under strict clearance demands. For the solution of this problem it was assumed that the chronological sequence, corresponding to the erection stage set, might be thought of as a set of independent sub-structures, each corresponding to an erection stage. This is done automatically by the mesh and variable linking generator. The number of RHS will be usually that of the final structure, due to load combination involving wind, earthquake and live load, acting in the several positions of the span.

\section{Reliability-based Optimization}

A failure event may be described by a functional relation, the limit state function in the following way

$$
\mathrm{F}=\{\mathrm{g}(\mathrm{x} \backslash 0\}
$$

The probability of failure may be determined by the following integral:

Where $f_{x}(x)$ is the joint probability density function of the random variables $X$. This integral is however non trivial to solve. Various methods for the solution have been proposed including numerical integration techniques, Monte Carlo simulation and asymptotic Laplace expansions. Numerical integration techniques become inefficient for increasing dimensions of the vector X. Monte Carlo simulation techniques may be used, but in the following the focus will be on the first order second moment methods (FORM) which are consistent with the solutions obtained by asymptotic Laplace integral expansions. In the case the limit state function $\mathrm{g}(\mathrm{x})$ is a linear function of the normally distributed basic random variables $X$ the probability of failure can be written in terms of the linear safety margin $M$ as:

$$
\mathrm{P}_{\mathrm{F}}=\mathrm{P}\{\mathrm{g}(\mathrm{x} \leq 0\}=\mathrm{P}(\mathrm{M} \leq 0)
$$

which reduces to the evaluation of the standard normal distribution function

$$
\mathrm{P}_{\mathrm{F}}=\Phi(-\beta)
$$

Where $\beta$ is the reliability index given as 
The reliability index has the geometrical interpretation as the smallest distance from the line (or the hyperplane) forming the boundary between the safe domain and the failure domain. The evaluation of the probability of failure reduces to simple evaluations in terms of mean values and standard deviations of the basic random variables.

When the limit state function is not linear in the random variables $\mathbf{X}$. Hasofer and Lind suggested to perform the linearization of the limit state function in the design point of the failure surface represented in normalized space $\mathbf{U}$.

$$
\mathrm{U}_{\mathrm{i}}=\left(\mathrm{X} \mathrm{i}-\mu_{\mathrm{xi}}\right) / \sigma_{\mathrm{xi}}
$$

As one does not know the design point in advance, this has to be found iteratively in a number of different ways. Provided that the limit state function is differentiable, the following simple iteration scheme may be followed:

$$
\begin{gathered}
\alpha_{i}=-\partial g(\beta \alpha) / \partial u_{i}\left[\sum_{j=1}^{n} \partial g(\beta \alpha)^{2} / \partial u_{i}\right]^{-1 / 2} \\
G\left(\beta \alpha_{1}, \beta \alpha_{2}, \ldots \beta \alpha_{v}\right)=0
\end{gathered}
$$

Which will provide the design point $\mathrm{u}^{*}$ as well as the reliability index $\beta$.

The reliability assessment requires an enumeration of the reliability indices associated with limit state functions to evaluate the structural system probability of failure. Collapse modes are usually correlated through loading and resistances, so an exact evaluation of the probability is impractical, or even impossible to perform numerically. For this reason, several investigators considered this problem by either finding bounds for $\mathrm{p}_{\mathrm{F}}$ or approximate solutions. In general, the admissible failure probability for structural design is very low. A first estimate of $\mathrm{P}_{\mathrm{f}}$ can be found through well-known first-order bounds proposed by Cornell:

$$
\underset{\text { allk }}{\operatorname{Max}}\left[\operatorname{Pr}\left(Z_{k)}\right] \leq p_{F} \leq \sum_{k=1, m} \operatorname{Pr}\left[Z_{k} \leq 0\right]\right.
$$

The lower bound, which represents the probability of occurrence of the most critical mode (dominant mode) is obtained by assuming the mode failure events $Z_{k}$ to be perfectly dependent, and the upper bound is derived by assuming independence between mode failure events. Hence, approximation by Cornell's first-order upper bound is very conservative because it neglects the high correlation between failure modes. Improved bounds can be obtained by taking into account the probabilities of joint failure events such as $\mathrm{P}\left(\mathrm{F}_{\mathrm{i}} \cap \mathrm{F}_{\mathrm{j}}\right)$ which means the probability that both events $F_{i}$ and $F_{j}$ will simultaneously occur. The resulting closed-form solutions for the lower and upper bounds are as follows:

$$
\begin{gathered}
p_{F} \geq\left(F_{1}\right)+\sum_{i=2}^{m} \operatorname{Max}\left\{\left[P\left(F_{i}\right)-\sum_{j=1}^{i-1} P\left(F_{i} \cap F_{j}\right] ; 0\right\}\right. \\
p_{F} \leq \sum_{i=1}^{m} P\left(F_{i}\right)-\sum_{i=2}^{m} \underset{j<i}{\operatorname{Max}} P\left(F_{i} \cap F_{j}\right)
\end{gathered}
$$

The above bounds can be further approximated using Ditlevsen's method of conditional bounding to find the probabilities of the joint events. This is accomplished by using a Gaussian distribution space in which it is always possible to determine three numbers $\beta_{\mathrm{i}}, \beta_{\mathrm{j}}$ and the correlation coefficient $\rho_{\mathrm{ij}}$ for each pair of collapse modes $F_{\mathrm{i}}$ and $\mathrm{F}_{\mathrm{j}}$. Improved bounds can also be obtained by using Vanmarcke's concept of failure mode decomposition which takes into account the conditional probability that the $(\mathrm{i}-1)$ mode survives given that mode $\mathrm{i}$ occurs. By assuming that the probability of occurrence of the ith mode $P\left(F_{i}\right)=\phi\left(\beta_{i}\right)$ depends on $\beta_{i}$ only, the conditional probability $P\left(S_{j} \mid F_{i}\right)$ is evaluated in terms of the safety indices $\beta_{\mathrm{i}}$ and $\beta_{\mathrm{j}}$ and the coefficient of correlation $\rho_{\mathrm{ij}}$ between the failure modes $\mathrm{F}_{\mathrm{i}}$ and $\mathrm{F}_{\mathrm{j}}$. A different approximate method which avoids calculating conditional probabilities resulting from conditions leading to failure via pairs of failure modes is the PNET. This method also requires the evaluation of the coefficients of correlation between any two failure modes $\mathrm{i}$ and $\mathrm{j}$ and is based on the notion of demarcating correlation coefficient $\rho_{0}$ assuming those failure modes with high correlation $\left(\rho_{\mathrm{i}, \mathrm{j}} \geq \rho_{0}\right)$ to be perfectly correlated and those with low correlation $\left(\rho_{i, j}<\rho_{0}\right)$ to be statistically independent. This method is not very convenient because the solutions will be heavily dependent on the assumed demarcating coefficient $\rho_{0}$. A discrete reliability sensitivity analysis is derived and used in the optimization algorithm

\section{Sensitivity analysis}

The analytic direct method was adopted for the purpose of sensitivity analysis, given the availability of the code, the discrete structural pattern and the large number of constraints under control. For ordinary linear statics problems, derivatives of kinematic constraints (displacements) are provided by solving a structural system with pseudo-loading. The stress derivatives are accurately determined from the chain derivation of the finite element stress matrix: 


$$
\begin{gathered}
\underline{\sigma}=\underline{D} \underline{B} e \underline{u} e \\
\frac{\partial \underline{\sigma}}{\partial \mathrm{x}_{\mathrm{i}}}=\frac{\partial(\underline{D} \underline{B} \underline{\mathrm{e}})}{\partial \mathrm{x}_{\mathrm{i}}} \underline{u_{\mathrm{e}}}+\underline{D} \underline{B} \underline{\mathrm{e}} \frac{\partial \underline{u_{\mathrm{e}}}}{\partial \mathrm{x}_{\mathrm{i}}}
\end{gathered}
$$

The first term of right-hand side may be directly computed during the computation of element contribution for the global system, on the condition that derivative expressions are pre-programmed and called on that stage. The second term on the right-hand side is somewhat more difficult to compute because an explicit relation between displacement vector and design variable set does not exist. Pre-programming and storing the stiffness matrix and right-hand side derivatives in the same way as described for the stress matrix, the displacement derivatives may be computed by the solution of $\mathrm{N}$ pseudo-load right-hand sides. The stress derivatives are then computed in a straightforward way. The explicit form of matrix derivatives depends on the type of element. For 2D and 3D bar and beam elements their calculation is a straightforward task. For plate-membrane elements, the differentiation of the whole finite element formulation is required.

\section{Optimization}

Pareto's economic principle is gaining increasing acceptance to multi-objective optimization problems. In minimization problems a solution vector is said to be Pareto optimal if no other feasible vector exists that could decrease one objective function without increasing at least another one. The optimum vector usually exists in practical problems and is not unique. In regard to reliability based design several alternative formulations exist. A comprehensive review can be found in 4 . The material cost together with maximum probability of failure and measures of the structural performance and imposed by manufacturing and technical considerations are the objectives to be minimized. Size, shape, material configuration and loading parameters may be allowed to vary during the optimization process. Bounds must be set for average cross-sectional and geometric design variables variables in order to achieve executable solutions and required aesthetic characteristics. The overall objective of cable-stayed bridges design is to achieve an economic and yet safe solution. In this study it is not intended to include all factors influencing the design economics. One of the factors conventionally adopted is the cost of material used. A second set of goals arises from the requirement that the stresses should be as small as possible. The optimization method requires that all these goals should be cast in a normalized form. Another set of goals arises from the imposition of lower and upper limits on the sizing variables, namely minimum cable cross sections to prevent topology changes and exequible dimensions for the stiffness girder and pylons cross sections: Similar bounds must be considered for the geometric design variables. Additional bounds are set when geometric design variables are considered, to ensure that no geometry violation occurs when these design variables are updated. Additional goals may be established in order to ensure the desired geometric requirements during the optimization process (mesh discretization, ratios of variation of cable spacing on deck and pylons, etc). For these the chosen approach was to initially supply all the necessary information, by means of a geometry coefficients set describing such conditions.

The objective is to minimize all of these objectives over sizing and geometry variables $\underline{\mathbf{X}}$. This problem is discontinuous and non-differentiable and is therefore hard to solve. However, by using an entropy-based approach, Templeman has shown that its solution is equivalent to that of an unconstrained convex scalar function, depending only on one control parameter, which may be solved by conventional quasi-Newton methods. This parameter must be steadily increased through the optimization process. The scalar function is very similar to that of Kreisselmeyer-Stainhauser, derived for control problems:

$$
\mathrm{F}(\underline{x})=\frac{1}{\rho} \cdot \ln \left[\sum_{\mathrm{j}=1}^{\mathrm{M}} \mathrm{e}^{\rho(\mathrm{g}(\underline{x}))}\right]
$$

Problem (13) is unconstrained and differentiable which, in theory, gives a wide choice of possible numerical solution methods. However, since the goal functions $\mathrm{g}_{\mathrm{j}}(\mathrm{x}, \mathrm{z})$ do not have explicit algebraic form in most cases, the strategy adopted was to solve (13) by means of an iterative sequence of explicit approximation models. An explicit approximation can be formulated by taking Taylor series expansions of all the goal functions $\mathrm{g}_{\mathrm{j}}(\mathrm{x}, \mathrm{z})$ truncated after the linear term. This gives:

$$
\left.\operatorname{Min} \mathrm{F}(\underline{x})=\frac{1}{\rho} \cdot \ln \left[\sum_{\mathrm{j}=1}^{\mathrm{M}} \mathrm{e}^{\rho\left(\mathrm{go}(\underline{\underline{x}})+\sum_{i=1}^{N} \frac{\partial \mathrm{goj}(\underline{x})}{\partial \mathrm{xi}^{\prime}} \mathrm{dxi}\right.}\right)\right]
$$


where $\mathrm{N}$ and $\mathrm{M}$ are respectively the number of sizing plus geometric design variables and the number of goal functions. $g_{o j}$ and $\partial g_{o j} / \partial x_{i}$ are the goals and their derivatives evaluated for the current design variable vector $\left(x_{0}, z_{o}\right)$, at which the Taylor series expansion is made.

Solving (14) for particular numerical values of $\mathrm{g}_{\mathrm{oj}}$ forms only one iteration of the complete solution of problem (13). The solution vector $\left(\mathrm{x}_{1}, \mathrm{z}_{1}\right)$ of such an iteration represents a new design which must be analysed and gives new values for $g_{1 j}, \partial g_{1 j} / \partial x_{i}$ and $\left(x_{1}, z_{1}\right)$, to replace those corresponding to $\left(x_{0}, z_{0}\right)$ in (14). Iterations continue until changes in the design variables become small. During these iterations the control parameter $\rho$ must not be decreased to ensure that a multiobjective solution is found.

\section{Numerical Example}

In order to illustrate the possibilities of the method, a numeric example is presented in this section. The starting trial design is as shown in Figure 3 and corresponds to an asymmetric two-span cable-stayed footbridge.

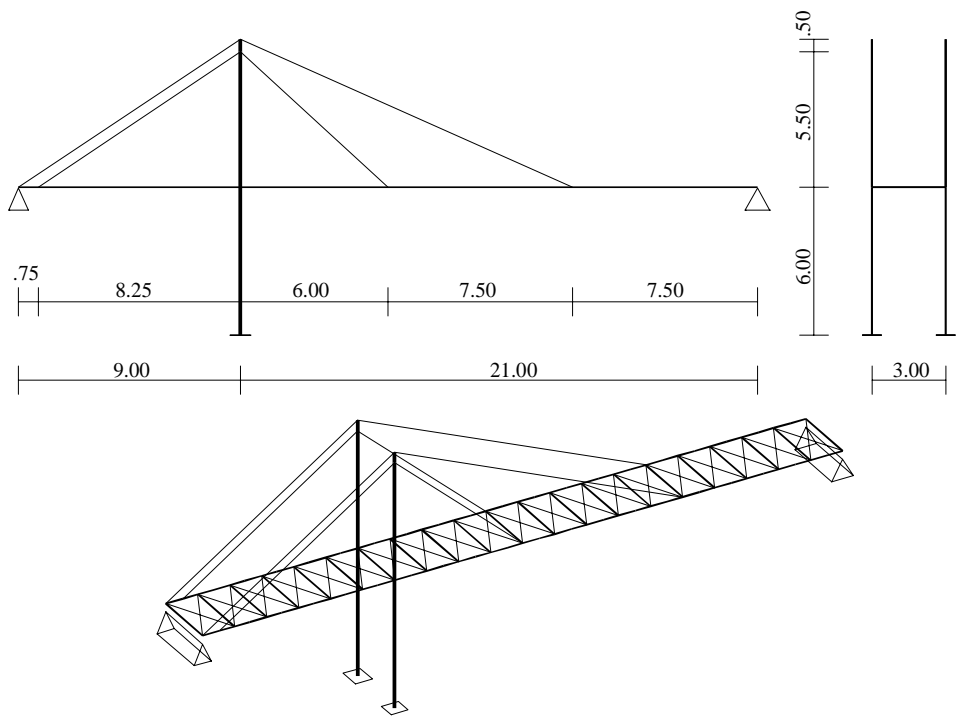

Figure 3 - Geometry of the bridge model - Starting trial design

28 design variables are considered of both sizing, shape and mechanical types. The former are those shown in Figure 4 and concern to cross-sectional dimensions for glulam beams and steel pylons and cross-sectional areas for the stays. Shape design variables are as illustrated in Figure 2, while mechanical design variables are the fixed-end prestressing forces and Young modulus for glued laminated timber. Starting values of shape design variables are given in Table 1 and their meaning is drawn from Figures 2 and 3.

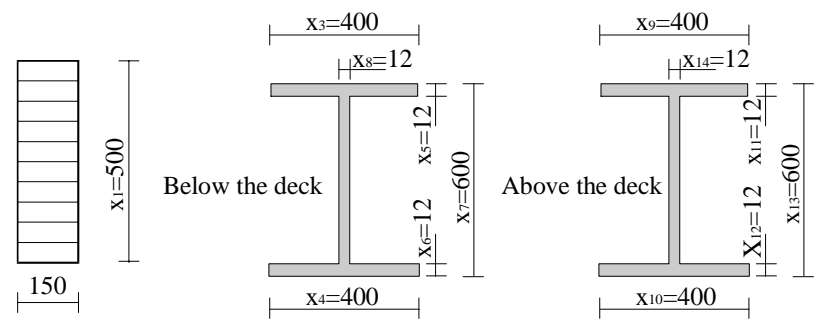

Figure 4 - Starting values of sizing design variables for the deck and pylons

Stiffening girders are made up of strength graded GL24h glulam. The mechanical characteristics are set according to EN1194 [7]. The fact that compressive strength is higher than tensile strength also favours the use of this material in cable-stayed bridges, because compression is the dominating state of stress in this structural system. A fixed width of $150 \mathrm{~mm}$ is considered, while the cross section depth is assigned to design variable \#1. Given the comparatively thin depth of the laminations, it is assumed to be possible a continuous variation of this parameter. The same assumption is made for Young modulus, which is assigned to design variable \#2. If the optimum design leads to a value of $E_{0, \text { mean, }}$ not corresponding to one of the glulam grades of prEN1194, an enumerative procedure may be used for the closest grades. This was not the case in the examples presented here, because the algorithm gives an optimum solution made up of 
GL20h. The sensitivity analysis concerning this type of design variable must account for the change in parameters that direct or indirectly affect the structural cost: the characteristic strength, the mass and the glulam cost itself. If the improvement of strength, associated to a variation of the Young modulus, is large enough to compensate the increase of structural self-weight and of more expensive glulam beams, the algorithm will lead to a superior quality material. For this relation to be properly accounted in the process, approximate relations between Young modulus and compressive strength, mass and cost were assumed. These are as follows:

$$
\begin{aligned}
& f_{c, 0, g, k}=2.15 E_{0, \text { mean }, g} \\
& \rho_{g, k}=0.16+0.02 E_{0, \text { mean }, g} \\
& €=€ 50\left(E_{0, \text { mean }, g}-1\right)
\end{aligned}
$$

with $f_{c, 0, g, k}$ expressed in $\mathrm{MPa}, E_{0, \text { mean }, g}$ in Gpa, $\rho_{g, k}$ in tons and the cost in Euro€.

Relations (15) and (16) lead to values slightly different from those specified in prEN1194, but the error is not large enough to change the trend towards the decrease or increase of the Young modulus. Equation (17) is an estimate which can be rewritten for a specific country and market conditions. The essential goal of this study is to find automatically a reliable solution in agreement with the available data.

A volume unit cost of $€ 10,000$ was prescribed for mild steel and $€ 25,000$ for cable steel.

Pylons are assumed to be made up with welded steel asymmetric I-shaped cross sections, the web plan being parallel to the longitudinal plan of the bridge. A fictitious lattice girder simulates the horizontal stiffness provided by the deck surface and required for transverse or eccentric loading.

All stays are assigned a starting cross-sectional area of $3 \mathrm{~cm}^{2}$ (design variables 15-18) and a fixed-end prestressing force of 50KN (design variables 19-22). It must be remarked that these are the tensile forces necessary to connect the stays to the anchorage devices in the undeflected structure and not those remaining in the cables after removal of the tensioning equipment, which are smaller due to the cable deformation recovery.

An uniform live load acting on the deck, with the characteristic value of $4 \mathrm{KN} / \mathrm{m}^{2}$, was considered as the leading action. It was considered it to act throughout the whole span or in either left or right span only. For the sake of simplicity, each of these live load distributions was assumed to be an independent event. Structural self-weight and a uniform load of $0.5 \mathrm{KN} / \mathrm{m}^{2}$ on deck were prescribed as dead loads. The EC1 [8] rules were used to define three load combinations for the purpose of ultimate strength limit state. These correspond to live load acting throughout the whole span or in each side ot the pylon only. Coefficients of variation (COV) for the dead and live loads were assigned the values of 0,10 and 0,20 , respectively. The corresponding safety factors for ultimate limit state design were 1,35 and 1,50. Given its irrelevancy for the issues focused in this paper, no strength reducing factor $k_{\text {mod }}$ [9] was considered.

Prescriptions of ENV1995-2 (EC5-Part 2: Bridges) [10] constraining the values of horizontal and vertical frequencies of the bridge were not included as well, because they are currently under implementation in the code. The main problem concerning this issue is the need for using the general procedure described in 7.2.1(5), because the cable-to-deck connection prevents the use of the simplified models of Table 7.1.

A gaussian probability density function with a coefficient of variation of $15 \%$ was assumed for the glued-laminated timber. Consistent with the traditional ultimate limit state design (level 1 approach), a design bending strength of $f_{m, g, d}=18,5 \mathrm{MPa}$ was used for the glulam elements. Fe510 was considered for the pylons and a high grade steel with $f_{s, y, d}=700 \mathrm{MPa}$ was used for the stay elements. With the usual safety factor of 1,30 for structural timber and the assumed coefficient of variation, these correspond to mean values of 31,9Mpa. Given the controlled production conditions and for the sake of simplicity, $\mathrm{COV}=0$ was generally adopted for the steel, though a range of values was tested in the parametric study which led to Figure 5.

Shape and sizing parameters referred to in Table 1 were also initially considered as random variables, with coefficients of variation of 0.01 . However, the results have shown no significant differences from the situation in which these parameters were considered as deterministic. Given the strong correlation between Young modulus and bending strength, here assumed as totally correlated, no simultaneous randomness was allowed for these two parameters for the reliability index evaluation.

In this example, the probability of failure concerns critical stresses throughout the structure, induced by the loadings. However, other failure modes or criteria could be used as well, such as the excessive deflection or cable understressing. The initial design was optimized through the use of the multicriteria approach described in section 6 to compare the reliability index and bimodal bounds in both cases. Starting and optimized values of design variables are listed in Table 1. The overall achieved cost reduction was about 39\%. For the starting design, a minimum reliability index of $\beta=3.47$, with an associate failure probability of $\Phi(-\beta)=2.6 \mathrm{E}-04$ and second order bounds of $2.55 \mathrm{E}-04 \leq \mathrm{P}_{\mathrm{f}} \leq 2.57 \mathrm{E}-04$ were found. The values for the optimized solution were $\beta=4.14, \Phi(-\beta)=1.75 \mathrm{E}-05$ and $1.75 \mathrm{E}-06 \leq \mathrm{Pf} \leq 3.55 \mathrm{E}-05$. The bound interval show that most of the nearly 1000 limit states considered are highly correlated. 
A parametric analysis was also conducted to evaluate the influence of the various random parameters involved. Figure 5 summarizes these results.

\begin{tabular}{|c|c|c|c|c|c|}
\hline DV & Starting Value & Optimized Value & DV & Starting Value & Optimized Value \\
\hline 1 & .49500 & .54349 & 2 & 11.00000 & 10.00000 \\
\hline 3 & .40000 & .20000 & 4 & .40000 & .20000 \\
\hline 5 & .01200 & .01000 & 6 & .01200 & .01000 \\
\hline 7 & .60000 & .30000 & 8 & .01200 & .01000 \\
\hline 9 & .40000 & .20000 & 10 & .40000 & .20000 \\
\hline 11 & .01200 & .01000 & 12 & .01200 & .01000 \\
\hline 13 & .50000 & .30000 & 14 & .01200 & .01000 \\
\hline 15 & .00030 & .00042 & 16 & .00030 & .00015 \\
\hline 17 & .00030 & .00047 & 18 & .00030 & .00010 \\
\hline 19 & 50.00000 & 245.61128 & 20 & 50.00000 & 88.70785 \\
\hline 21 & 50.00000 & 189.93720 & 22 & 50.00000 & 39.50334 \\
\hline 23 & .75000 & 1.54763 & 24 & .50000 & .30000 \\
\hline 25 & 7.50000 & 5.25753 & 26 & 7.50000 & 5.64119 \\
\hline 27 & 5.50000 & 4.00000 & 28 & 9.00000 & 9.36154 \\
\hline
\end{tabular}

Table 1 - Initial and final (optimized) values of design variables

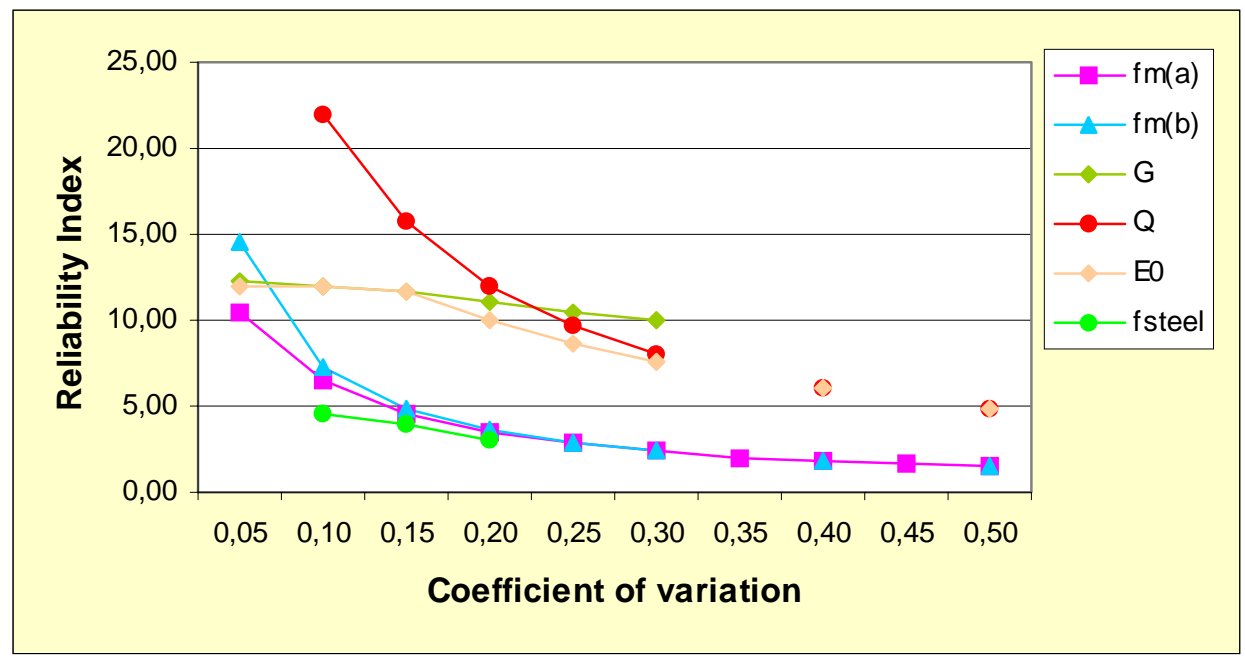

Figure 5 - Parametric analysis on the influence of various random parameters

\section{References}

1. Negrão, J.H.O. and Simões, L.M.C. Optimization of Cable-stayed bridges with 3-dimensional modelling, Computers \& Structures, 1997, 64:741-758

2. Negrão, J.H.O. and Simões, L.M.C., Shape and sizing optimisation of box-girder decks of cable-stayed bridges, Proc. OPTI 99 (Sixth Int. Conf. on Computer Aided Optimum Design of Structures), edts. S.Hernandez, A.J.Kassab \& C.A. Brebbia, WITpress, 1999, 323-332.

3 Simões, L.M.C. and Negrão, J.H.O. Optimization of Cable-stayed bridges subjected to earthquakes with non-linear behaviour, Engineering Optimization, 1999, 31:457-478

4. Thoft-Christensen, P. On Reliability-based Structural Optimization in A.Der Kiureghian and P. Thoft Christensen, Ed. Reliability and Optimization of Structural Systems, Proc. $3^{\text {rd }}$ WG 7.5 IFIP Conf. Berkeley 26-28 March 1990, Berlin, 1991, 387-402 